\title{
Chumbo: Uma introdução à extração e a fitorremediação
}

\section{Lead: An introduction to extraction and phytoremediation}

\author{
Juliana Cristina Holzbach ${ }^{1,2}$, Edna Isabela Teobaldo Marques Barros ${ }^{2}$, Maike de Oliveira \\ Krauser $^{1}$ e Paulo Vitor Brandão Leal ${ }^{*}$ \\ ${ }^{1}$ Química Ambiental; Universidade Federal do Tocantins; 77410-530; Gurupi - TO - Brasil, ${ }^{2}$ Farmácia; Centro \\ Universitário Unirg; 77403-090; Gurupi - TO - Brasil.
}

\begin{abstract}
Lead is a commonly used metal in modern industry, because of its useful physico-chemical properties, for instance, to application in eletronic equipment. However, the major concern in the use of the current metal refers to the irregular drop, since $40 \%$ in the equipment weight corresponds to the lead. The lead, being a heavy metal, fits between the more toxic elements to the environment, and especially to human beings. The lead contamination is a concern and nowadays researchers are developing Phytoremediation methods for the decontamination of these polluted areas. Phytoextraction is the most recommended technique for heavy metals extraction. Thus this paper aims to make a survey of the main uses and the impacts of the lead on living beings, besides studying the application of the method of Phytoremediation in contaminated areas by this metal.
\end{abstract}

Key-words: Lead, contamination, phytoremediation

\section{INTRODUÇÃO}

\section{Do minério a aplicação}

O chumbo é um metal branco azulado, com número atômico 82 , pertencente ao $5^{\circ}$ período da tabela periódica e ao grupo do carbono (Grupo 14). As características deste fazem com que ele seja um dos mais importantes metais desde a revolução industrial (Atkins, 2006).
Por se apresentar em estado nativo na crosta terrestre e por apresentar propriedades físicas peculiares, tabela 1 , este metal vem sendo utilizado pelos humanos desde pelo menos 7000 a.C., a sua baixa temperatura de fusão e abundância facilitaram a sua utilização em tempos antigos (Instituto de Física-UFRJ, 2012).

Tabela 1. Propriedades físicas do chumbo (ADAPTADO DE: Museu de Minerais e Rochas "Heinz Ebert"-Unesp, 2012).

\begin{tabular}{ll}
\hline Estado Natural & Sólido \\
\hline Cor & Branco-Azulado \\
Ponto de Fusão & $327,46{ }^{\circ} \mathrm{C}$ \\
Ponto de Ebulição & $1748,85^{\circ} \mathrm{C}$ \\
Dureza & $2-2,5$ \\
Densidade Relativa & $11,35 \mathrm{~kg} / \mathrm{dm}^{3}$ \\
Propriedades Diagnósticas & Mole e Maleável \\
\hline
\end{tabular}

A peça mais antiga de chumbo até hoje descoberta, data de 3800 a.C.. e está em exposição no Museu Britânico. Há ainda evidências da utilização deste metal pelos chineses e pelos povos romanos em encanamentos feitos de chumbo, utilizados até hoje, que são datados de 300 a.C., (Instituto de Física-UFRJ, 2012). O chumbo pode ser obtido em seu estado natural, no entanto, sua abundância é baixa em comparação a outros minerais. Os íons derivados de chumbo podem estar associados a

\footnotetext{
$\overline{\text { *Author for correspondence: pvitorufla@yahoo.com.br }}$
} 
mais de 60 tipos distintos de minerais, sendo os minerais mais importantes e suas composições descritas na tabela 2 (Museu de Minerais e Rochas "Heinz Ebert"- Unesp, 2012).

Tabela 2. Composição dos minerais mais importantes associados ao chumbo (ADAPTADO DE: Museu de Minerais e Rochas "Heinz Ebert"- Unesp, 2012).

\begin{tabular}{|c|c|c|}
\hline Mineral & Composição & Associação \\
\hline Galena & $\mathrm{PbS}$ & $\mathrm{Zn}, \mathrm{Ag}, \mathrm{Cu}, \mathrm{Fe}$ dentro outros \\
\hline Cerussita & $\mathrm{PbCO}_{3}$ & Galena, Esfarelita e minerais secundários \\
\hline Anglessita & $\mathrm{PbSO}_{4}$ & Galena \\
\hline Boulangerita & $5 \mathrm{PbS} .2 \mathrm{Sb}_{2} \mathrm{~S}_{3}$ & Galena e Sb \\
\hline Bournonita & $2 \mathrm{PbS} . \mathrm{Cu}_{2} \mathrm{~S} \mathrm{Sb}_{2} \mathrm{~S}_{3}$ & Galena, $\mathrm{Cu}$ e $\mathrm{Sb}$ \\
\hline Piromorfita & $\mathrm{Pb}_{5}\left(\mathrm{PO}_{4}\right)_{3} \mathrm{Cl}$ & Galena e Cerussita \\
\hline Jamesonita & $\mathrm{Pb}_{4} \mathrm{FeSb}_{6} \mathrm{~S}_{14}$ & Minérios de $\mathrm{Zn}$ e $\mathrm{Cu}$ \\
\hline Wulffenita & $\mathrm{PbMnO}_{4}$ & Outros molibdatos \\
\hline
\end{tabular}

A obtenção de chumbo é feita principalmente a partir do mineral Galena, por apresentar maior concentração do elemento, por volta de $87 \%$. Na sua obtenção é utilizado o método de Ustulação. Neste método, inicialmente se aquece o mineral com fluxo de oxigênio obtendo óxido de chumbo como segue a reação (Rocha, 1973):

$$
\mathrm{PbS}+\mathrm{O}_{2} \rightarrow \mathrm{PbO}+\mathrm{SO}_{2}
$$

Após a obtenção do óxido de chumbo (PbO), um dos métodos utilizados para a formação do chumbo metálico é adicionar o óxido de chumbo em um alto forno juntamente com agentes redutores. O chumbo obtido é separado por flotação e purificado por destilação, o chumbo nesta etapa apresenta pureza de 99,99\% (Rocha, 1973).

Devido as suas características, o chumbo, apresenta uma diversidade de potenciais de aplicação, dentre estas, pode-se destacar a utilização como protetor radiológico, pois, apresenta uma alta densidade, absorvendo desta maneira radiação ionizante; na indústria de automóvel, onde é aplicado na confecção de baterias automotivas e também no balanceamento dos pneus (Bocchi et al., 2000).

Uma aplicação que vem sendo motivo de preocupação na sociedade moderna é a sua utilização na indústria de eletrônicos, onde é empregado em soldas e em tubos de raios catódicos, pois o descarte destes materiais muitas vezes é realizado de maneira inadequada. Estimase que e se estima que $40 \%$ da massa dos equipamentos eletroeletrônicos são constituídas por chumbo (Oliveira et al., 2010).
$\mathrm{O}$ alto emprego industrial deste metal se deve a resistência à corrosão. Quando recém cortado apresenta um brilho metálico que desaparece em contato com o ar. Isso se deve a formação do óxido de chumbo, formando assim um revestimento inerte que o protege (Rocha, 1973). Esta característica faz com que este metal seja empregado no revestimento de cabos, tubulação em indústrias, transporte de reagentes oxidantes, na fabricação de vidros dentre outros (Alves et al., 2001; Duarte e Pasqual, 2000; Mardones, 2007).

$\mathrm{O}$ chumbo é considerado um metal pesado indiferente das definições utilizadas para esta designação, dentre as principais definições tem-se a classificação de metal pesado apenas para elementos que apresentam massa específica acima de $3,5 \mathrm{~g} . \mathrm{cm}^{-3}$, sendo o chumbo um metal com massa específica maior que $10,0 \mathrm{~g} \cdot \mathrm{cm}^{-3}$. Outras definições de metal pesado consideram a massa atômica, sendo o sódio (massa atômica 23) dado como referência, ou consideram o número atômico, sendo o cálcio (número atômico 20) como referência (Lima e Merçon, 2011).

De acordo com a CERCLA (Comprehensive Environmental Response, Compensation, and Liability Act) e a OMS (Organização Mundial da Saúde) existem dez substâncias ameaçadoras ao organismo humano, dentre elas quatro são metais pesados, onde pode-se destacar como os mais tóxicos o chumbo, mercúrio e o arsênio (Azevedo, 2010). Entre os inúmeros compostos que contém o chumbo podemos enfatizar duas classes, consideradas diferentes de acordo com a toxicidade (Schifer et al., 2005; Rodrigues, 2008).

\section{CLASSIFICAÇÃO E PATOLOGIAS}




\section{Chumbo inorgânico}

Esta classe pode afetar inúmeros sistemas, com isso provoca numerosas patologias e alterações clínica, tendo ação preferencial pelos ossos. A absorção do chumbo inorgânico depende do tempo de exposição, da intensidade da exposição, bem como fatores vinculados ao indivíduo (Moreira e Moreira, 2004, Moreira e Moreira, 2004, Moreira e Moreira, 2004).

\section{Chumbo orgânico}

Acontece pelo envenenamento do chumbo tetrametila e tetraetila (Figura 1). São solúveis em gordura, ou seja, lipossolúveis e são fáceis de ser absorvidos, chegando a causar distúrbios neurológicos (Teixeira et al., 2004).
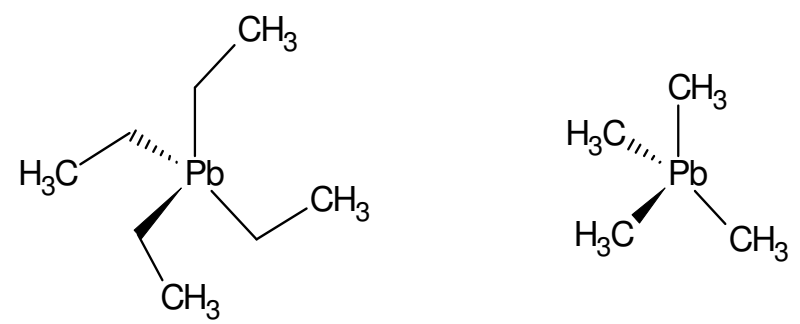

Figura 1- Estrutura do chumbo tetraetila e do chumbo tetrametila.

Devido a esta alta toxicidade, seu descarte assim como sua manufatura devem ser realizados com os devidos cuidados e responsabilidades. Em nosso país existem leis que estabelecem limites de tolerância para o chumbo. A Portaria ${ }^{\circ} 685$ de 27 de agosto de 1998 da ANVISA (Agência Nacional de Vigilância Sanitária) impõem limites máximos de tolerância de contaminantes inorgânicos em alimentos, sendo o máximo permitido de chumbo inorgânico em determinados alimentos de até 2,0 mg. $\mathrm{kg}^{-1}$.

A portaria n 24 da Secretaria de Seg e Saúde através da Norma Reg n 7 de 29 de dezembro de 1994 instituiu que o valor adequado de chumbo no organismo humano deve estar entre o limite de 40 $-60 \mu \mathrm{g}$ de chumbo para cada $100 \mathrm{~mL}$ de sangue, acima deste valor é considerado exposição excessiva ao chumbo (Brasil, 1994; Araújo et al., 1999; Jacob et al., 2002).

A intoxicação pelo chumbo acontece lenta e gradativamente devido sua capacidade de se acumular no organismo (Sadao, 2002). Tendo em vista esta capacidade de acumulação, por questões de saúde pública se faz necessária à substituição de chumbo por compostos menos agressivos.
Podem-se destacar, por exemplo, a reformulação da gasolina pela adição de compostos aromáticos ao invés do chumbo como agente antidetonante e a substituição de pigmentos a base deste metal em tintas empregando-se, em alguns casos, o hidróxido de ítrio (Spiro e Stigliani, 2008).

Denomina-se saturnismo ou intoxicação por chumbo a moléstia provocada pela exposição a este metal. Esta enfermidade pode ter ascendência no ambiente natural ou no ambiente de trabalho. Por exemplo, comunidades que moram próximas a indústrias têm, consequentemente, uma concentração maior de chumbo no sangue quando comparadas a comunidades mais afastadas (Brasil, 2001; Neme et al., 2009).

A alta concentração de chumbo no sangue denominado plumbemia - afeta significativamente o sistema nervoso das crianças, acarretando dificuldade de aprendizado, dores de cabeça frequentes e redução da visão (Dascanio et al., 2010).

O chumbo compromete determinados sistemas como o sistema hematológico, o sistema digestivo, renal, neuromuscular, endócrino, circulatório, reprodutivo, sistema nervoso central e periférico. Este comprometimento pode ter efeitos imediatos ou tardios. Em exemplo aos efeitos imediatos podemos citar as consequências sobre o sistema nervoso e ao sistema hematopoiético que ocorre logo após a exposição excessiva ao chumbo (Troster, 1993; Mattos et al., 2003; Cavalcante, 2009).

No tratamento da intoxicação por chumbo na sua fase aguda faz-se necessárias medidas preventivas bem como constantes orientações para não ocorrer intoxicações posteriores. Deve-se realizar exames para a averiguação da concentração deste metal no sangue e, quando necessário, começar o tratamento por quelantes (Schifer et al., 2005).

A contaminação por chumbo não afeta somente os seres humanos presentes nas áreas poluídas, mas todo o bioma contaminado pela presença do metal.

\section{Fitorremediação}

Uma área de pesquisa que está em constante desenvolvimento e expansão que abrange as possíveis maneiras de descontaminação de áreas poluídas é a fitorremediação, onde se empregam sistemas vegetais fotossintetizantes e sua microbiota como agentes remediadores (Barros et al., 2010).

Em solos contaminados por chumbo a Fitorremediação pode ser utilizada através de duas 
estratégias distintas: a Fitoestabilização ou a Fitoextração.

A Fitoestabilização utiliza de plantas para reduzir o perigo intrínseco do contaminante, absorvendoo, mas sem fazer a remoção do contaminante, deste modo se reduz a sua disponibilidade no ambiente contaminado. A Fitoextração, diferentemente da Fitoestabilização, remove o contaminante do meio. Este método utiliza de plantas para absorver o contaminante e posterior processamento. Hoje se apresenta como uma técnica atraente economicamente para a extração de metais como o chumbo (Huang e Cunningham, 1996).

Alves et al. (2008), descreve a utilização de plantas para a Fitoextração de chumbo, destacando a necessidade da alta tolerância da planta para o emprego como Fitorremediador.

$\mathrm{O}$ excesso de chumbo no meio pode resultar em diversos sintomas de toxicidade em plantas onde se destaca: redução do crescimento, inibição da fotossíntese, alteração do sistema mineral e o balanço hídrico dentre outros. Já se conhece na literatura os efeitos morfológicos, fisiológicos e bioquímicos da toxicidade das plantas assim como as estratégias utilizadas por estas em um desenvolvimento com tolerância ao chumbo (Dubey e Sharma, 2005).

A absorção de chumbo em culturas importantes para os seres humanos vem sendo estudadas, dentre estas destaca-se a cultura do milho. Merlino et al. (2010), apresenta em seu trabalho a absorção de metais pesados incluindo o chumbo no desenvolvimento do milho, quantificando a presença desses metais nas folhas e nos grãos. Em seu estudo observou-se que os teores de chumbo em grãos de milho permaneceram abaixo dos limites estabelecidos para o consumo humano, assim como de outros metais.

Uma técnica promissora utilizada na Fitoextração é a de formação de complexos metal-quelante, para auxiliar o potencial extrator da planta. Destaca-se, dentre os quelantes, o ácido etilenodiamino tetraacético (EDTA). No entanto, podem ser utilizados compostos naturais que apresentam maior velocidade de degradação, quando comparada a compostos sintéticos como o EDTA, por exemplo, ácidos cítricos e gálico (Melo, 2008).

Pereira et al. (2007), descreve a capacidade Fitoextratora de milho em Latossolos tratado com chumbo e EDTA, observando neste caso, que a
Fitoextração do milho não se recomendada a utilização de EDTA.

Em outro trabalho, Pereira et al. (2010), observou que o tratamento com EDTA em solos contendo chumbo aumentam o potencial Fitoextrator do feijão-de-porco (Canavalia ensiformis $L$.).

\section{RESUMO}

O chumbo é um dos metais mais utilizados na indústria moderna, pois apresenta propriedades físico-químicas úteis para sua aplicação em equipamentos eletroeletrônicos. Porém, uma das maiores preocupações atuais na utilização desse metal refere-se ao descarte irregular, uma vez que, cerca de $40 \%$ da massa de alguns equipamentos é composta por chumbo. $\mathrm{O}$ chumbo, além de ser um metal pesado, enquadra-se entre os elementos mais tóxicos para o meio ambiente e principalmente ao ser humano. A contaminação por chumbo é preocupante e hoje se estuda métodos de fitorremediação para a descontaminação destas zonas poluída sendo a fitoextração a mais recomendada para metais pesados. Desse modo o presente trabalho visa fazer um levantamento sobre as principais utilizações e os impactos gerados pelo chumbo aos seres vivos, além de estudar a aplicação do método de fitorremediação em zonas contaminadas por este metal.

Palavras-chave: Chumbo, contaminação, fitorremediação

\section{CONCLUSÕES}

A utilização do chumbo, como matéria prima, na indústria moderna é imprescindível, devido às propriedades físico-químicas apresentadas por este metal. Deste modo, os cuidados em sua manufatura, em sua manipulação nestas indústrias, assim como, em sua estocagem devem seguir regras meticulosas para que não ocorra contaminação de funcionários e/ou moradores em sua redondeza.

Hoje o Brasil ainda necessita de leis mais específicas sobre a manipulação deste metal, principalmente quanto ao seu uso como pigmentos para tinturas. Uma legislação eficiente deve incluir ainda, maneira específica para o descarte de materiais que contenham chumbo, enfatizando, principalmente, os equipamentos eletroeletrônicos, pois estes apresentam hoje um volume de descarte preocupante para o meio ambiente e consequentemente a saúde humana.

A Fitorremediação apresenta hoje uma alternativa promissora na descontaminação de áreas degradadas, sendo a Fitoextração uma técnica economicamente viável para ser aplicada em ambientais contaminados por chumbo, podendo 
ser utilizada juntamente com compostos quelantes para melhorar sua eficiência.

\section{REFERÊNCIAS}

Alves, J. C.; Souza, A. P.; Pôrto, M. L.; Arruda, J. A.; Júnior, U. A. T.; Silva, G. B.; Araújo, R. C.; Santos, D. (2008), Absorção e distribuição de chumbo em plantas de vetiver, jureminha e algaroba. Revista Brasileira de Ciências do Solo, 32, 1329-1336.

Alves, O. L.; Gimenez, I. F.; Mazali, I. O. (2001), Vidros, Química Nova na Escola, Edição Especial.

Araújo, U. C.; Pivetta, F. R.; Moreira, J. C. (1999), Avaliação da exposição ocupacional ao chumbo: proposta de uma estratégia de monitoramento para prevenção dos efeitos clínicos e subclínicos. Caderno de Saúde Pública, 15, 123-131.

Atkins, P.; Jones, L. (2006), Princípios de Química: Questionando a vida moderna e o meio ambiente. 3. Ed. Porto Alegre: Bookman.

Azevedo, M. F. A. (2010), Abordagem inicial no atendimento ambulatorial em distúrbios neurotoxicológicos. Parte I - metais. Revista Brasileira de Neurologia, 46, 17-31.

Barros, Y. J.; Melo, V. F.; Sautter, K. D.; Buschler, B.; Oliveira, E. D.; Azevedo, J. C. R.; Souza, L. C. P.; Kummer, L. (2010), Indicadores de qualidade de solos de área de mineração e metalurgica de chumbo. II - mesofauna e plantas, Revista Brasileira de Ciências do Solo, 34, 14131426.

Bocchi, N.; Ferracin, L. C.; Biaggio, S. R. (2000), Pilhas e Baterias: Funcionamento e impacto ambiental, Química Nova na Escola, 11, 3-9.

Brasil. Ministério da Saúde. NR-7 - Norma Regulamentadora de Segurança e Saúde no Trabalho. Redação dada pela Portaria n. 24, de 2912-1994/ DOU de 30/12/1994.

BRASIL. Ministério do Trabalho e Emprego. O chumbo e as formas de controle. Ministério do Trabalho e Emprego, FUNDACENTRO Fundação Jorge Duprat Figueiredo de Segurança e Medicina do Trabalho. Brasília. (2001), Disponivel em: <http://portal.mte.gov.br/portalmte/> Acesso: 18 de junho de 2012.
Capitani, E. M. (2009), Diagnóstico e tratamento da intoxicação por chumbo em crianças e adultos. In: Chumbo e a Saúde Humana, Simpósio, 42, 3, 319-329.

Carvalho, F. M.; Neto, A. M. S.; Tavares, T. M.; Costa, A. C. A.; Chaves, C. D.; Nascimento, L. D.; Reis, M. A. (2001), Chumbo no sangue de crianças e passivo ambiental de uma fundição de chumbo no Brasil. Revista Panamericana de Saúde Pública, 13, 19-24.

Cavalcante, A. L. M. Parâmetros sanguíneos de tilápia (Oreochromis niloticus) no monitoramento ambiental do chumbo. Dissertação (Mestrado em Ciências na área de Saúde Pública) - Fundação Oswaldo Cruz, 2009.

Dascanio, D.; Rodrigues, O. M. R; Valle, T. G. M. (2010), Relação entre os estilos parentais e o desempenho intelectual de crianças com plumbemia. Avaliação Psicológica, 9, 461-470.

Duarte, R. P. S. e Paqual, A. (2000), Avaliação do cádmio $(\mathrm{Cd})$, chumbo $(\mathrm{Pb})$, Niquel $(\mathrm{Ni})$ e zinco (Zn) em solos, plantas e cabelos humanos. Energia e Agricultura, 15, 46-58.

Dubey, R. S. e Sharma, P. (2005), Lead toxicity in plants, Brazilian Journal of Plant Physiology, 17, 35-52.

Huang, J. W. e Cunningham, S. D. (1996), Lead phytoextration: species variation is lead uptake and translocation. New Phytologist, 134, 75-84.

Instituto de Física, Universidade Federal do Rio de Janeiro. Disponível em: $<$ http://www.if.ufrj.br/teaching/elem/e08200.html $>$ Acesso em: 15 de junho de 2012.

Jacob, L. C. B.; Alvarengas, K. F.; Morata, T. C. (2002), Os efeitos da exposição ocupacional ao chumbo sobre o sistema auditivo: uma revisão da literatura. Revista Brasileira de Otorrinolaringologia, 68, 564-569.

Lima, V. F. e Merçon, F. (2011), Metais Pesados no Ensino de Química, Química Nova na Escola, 33, 199-205.

Mardones, C. G. (2007), O trabalhador e o princípio da dignidade humana: A utilização de 
metis pesados nas indústrias brasileiras e a ausência de legislação brasileira efetivamente protetora. Cadernos de Direito, 7, 25-40.

Mattos, U. A. O.; Fortes, J. D. N.; Shubo, A. M. R.; Portela, L. F.; Gómez, M. B.; Tabalippa, M.; Shubo, T. (2003), Avaliação e diagnóstico das condições de trabalho em duas indústrias de baterias chumbo-ácidas no estado do Rio de Janeiro. Ciência e Saúde Coletiva, 8, 1047-1056.

Melo, E. E. C.; Nascimento, C. W. A.; Accioly, A. M. A.; Santos, A. C. Q. (2008), Fitoextração e fracionamento de metais pesados em sopo após múltiplas aplicações de ácidos orgânicos, Scientia Agricola, 65, 61-68.

Merlino, L. C. S.; Melo, W. J.; Macedo, F. G.; Guedes, A. C. T. P.; Ribeiro, M. H.; Melo, V. P.; Melo, G. M. P. (2010), Bário, Cádmio, Cromo e Chumbo em plantas de milho e em latossolo após onze aplicações anuais de lodo de esgoto, Revista Brasileira de Ciências do Solo, 34, 2031-2039.

Moreira, F. R. e Moreira, J. C. (2004), A cinética do chumbo no organismo humano e sua importância para a saúde. Ciência \& Saúde Coletiva, 9, 167-181.

Moreira, F. R. e Moreira, J. C. (2004), A importância da análise de especiação do chumbo em plasma para a avaliação dos riscos a saúde. Química Nova, 27, 251-260.

Moreira, F. R. e Moreira, J. C. (2004), Os efeitos do chumbo sobre o organismo humano e seu significado para a saúde. Organización Panamericana de La Salud, 15, 119-129.

Museu de Minerais e Rochas "Henz Ebert", Universidade Estadual Paulista. Disponível em: $<$ http://www.rc.unesp.br/museudpm/> Acesso em: 15 de junho de 2012.

Neme, C. M. B.; Pereira, P. M.; Rodrigues, O. M. P. R.; Valle, T. G. M.; Melchiori, L. E. (2009), Indicadores de comprometimento emocional avaliadas por chumbo. Arquivos de Ciência $e$ Saúde, 16, 15-20.
Oliveira, R. S.; Gomes, E. S.; Afonso, J. C. (2010), O Lixo Eletrônico: Uma Abordagem para o Ensino Fundamental e Médio. Química Nova na Escola, 32, 240-248.

Pereira, B. F. F.; Abreu, C. A.; Herpin, U.; Abreu, M. F.; Berton, R. S. (2010), Phytoremediation of lead by jack bens on Rhodic Hapludox amended with EDTA. Scintia Agricola. 67, 308-318.

Pereira, B. F. F.; Abreu, C. A.; Romeiro, S.; Lagôa, A. M. M. A.; Paz-González, A. (2007), Fitoextração de $\mathrm{Pb}$ por milho em um Latossomo tratado com $\mathrm{Pb}$ e EDTA. Scientia Agricola, 64, 52-60.

Rocha, A. J. D. (1973), Perfil Analítico do Chumbo, Ministerio de Minas e Energia, Boletim $\mathrm{N}^{\mathrm{o}} 8$.

Rodrigues, L. C. V. Estudo da disponibilidade química de chumbo e arsênio em sedimentos de corrente e colunas de sedimentos no Vale do Ribeira - SP. Tese (Doutorado em Geoquímica Ambiental) - Universidade Federal Fluminense, 2008.

Sadao, M. (2002), Intoxicação por metais. Revista de Oxidologia, 37-42.

Schifer, T. S.; Bogusz, S.; Montano, M. A. E. (2005) Aspectos toxicológicos do chumbo. Infarma, 17, 67-71.

Spiro, T. G. e Stigliani, W. M. (2009) Química Ambiental. 2. Ed. São Paulo: Pearson Prentice Hall.

Teixeira, V. G.; Coutinho, F. M. B.; Gomes, A. S. (2004) Resinas poliméricas para a separação e préconcentração de chumbo. Química Nova, 27, 754762.

Troster, E. J. (1993), Intoxicação plúmbica, Departamento de Pediatria Universidade de São Paulo, 15, 45- 49. 